\title{
Three dimensional vibration analysis of an infinite poroelastic plate immersed in an inviscid elastic fluid
}

\author{
S. Ahmed Shah ${ }^{1} *$ M. Tajuddin ${ }^{2}$ \\ ${ }^{1 *}$ Department of Mathematics, Deccan College of Engineering and Technology, Hyderabad, INDIA \\ ${ }^{2}$ Department of Mathematics, Osmania University, Hyderabad, INDIA \\ "Corresponding Author: e-mail: ahmed_shah67@yahoo.com.
}

\begin{abstract}
Three dimensional wave propagation in poroelastic plate immersed in an inviscid elastic fluid is studied employing Biot's theory. Frequency equations are derived for pervious and impervious surfaces. Frequency equation each for a pervious and impervious surface is obtained for poroelastic plate in contact with an inviscid elastic fluid and poroelastic plate in vacuum as a particular case and also when the wavenumbers vanish. Phase velocity as a function of propagation constant is computed for pervious and impervious surfaces in each case, i.e., poroelastic plate immersed in an acoustic medium, poroelastic plate in contact with an inviscid elastic fluid and poroelastic plate in vacuum in absence of dissipation. It is observed that the phase velocity of pervious and impervious surfaces is same for water saturated sandstone while it is not for kerosene saturated sandstone in each of these three cases. Results of previous investigations are obtained as a particular case of the present study.
\end{abstract}

Keywords: Biot's theory, poroelastic plate, pervious surface, impervious surface, propagation constant, phase velocity, elastic fluid.

\section{Introduction}

Stress free waves in elastic bars of rectangular cross section are studied by Mindlin and Fox (1960). Walter and Anderson (1970) studied wave propagation in an infinite elastic plate in contact with an inviscid liquid layer. Account on loose bonding of elastic half-spaces was given by Banghar et al. (1976) along with different limiting cases. Gazis (1959) discussed the propagation of free harmonic waves along a hollow elastic circular cylinder of infinite extent and presented numerical results. Mindlin (1986) studied flexural vibrations of rectangular plates with free edges. Flexural vibrations of rectangular thin plates with free boundary conditions are studied by Shuyu (2001). Employing Biot's (1956) theory, Tajuddin and Ahmed (1991) studied the dynamic interaction of a poroelastic layer and half-space. Malla Reddy and Tajuddin (2003) solved the problem of edge waves in poroelastic plate under plane stress conditions. Kanj and Abousleiman (2004) presented poromechanical solutions of Lame' problem and discussed different cases in detail. Chao et al (2004) studied the shock-induced borehole waves in porous formations. Tajuddin and Shah $(2006,07,09,10(\mathrm{~b}, \mathrm{c}))$ studied different problems on wave propagation in poroelastic cylinders. Shah $(2008,10(a))$ investigated the axially symmetric vibrations of fluid-filled poroelastic circular cylindrical shells of various wallthicknesses and flexural vibrations of coated poroelastic cylinders of infinite extent in absence of dissipation.

In the present analysis, wave propagation in an infinite poroelastic plate submerged in an inviscid elastic fluid is studied in absence of dissipation. Frequency equation each for a pervious and an impervious surface is obtained employing Biot's (1956) theory of wave propagation in liquid saturated poroelastic solid. Biot's model consists of an elastic matrix permeated by a network of interconnected spaces saturated with liquid. Frequency equation each for a pervious and impervious surface is obtained for poroelastic plate in contact with an inviscid elastic fluid and poroelastic plate in vacuum as a particular case and also when the wavenumber vanishes.

Non-dimensional phase velocity as a function of propagation constant is computed for pervious and impervious surfaces in each case, i.e., poroelastic plate immersed in an inviscid elastic fluid, poroelastic plate in contact with an inviscid elastic fluid and poroelastic plate in vacuum in absence of dissipation. The results are presented graphically for two types of poroelastic materials 
and then discussed. It is observed that the phase velocity of pervious and impervious surfaces, in general, is same in case of water saturated sandstone while it not for kerosene saturated sandstone. Results of previous investigations are obtained as a particular case of the present study. Thus the present investigation is more generalized.

\section{Governing equations}

The equations of motion of a homogeneous, isotropic poroelastic solid (Biot, 1956) in presence of dissipation $b$ are

$$
\begin{array}{r}
\mathrm{N} \nabla^{2} \overrightarrow{\mathrm{u}}+(\mathrm{A}+\mathrm{N}) \nabla \mathrm{e}+\mathrm{Q} \nabla \in=\frac{\partial^{2}}{\partial \mathrm{t}^{2}}\left(\rho_{11} \overrightarrow{\mathrm{u}}+\rho_{12} \overrightarrow{\mathrm{U}}\right)+\mathrm{b} \frac{\partial}{\partial \mathrm{t}}(\overrightarrow{\mathrm{u}}-\overrightarrow{\mathrm{U}}), \\
\mathrm{Q} \nabla \mathrm{e}+\mathrm{R} \nabla \in=\frac{\partial^{2}}{\partial \mathrm{t}^{2}}\left(\rho_{12} \overrightarrow{\mathrm{u}}+\rho_{22} \overrightarrow{\mathrm{U}}\right)-\mathrm{b} \frac{\partial}{\partial \mathrm{t}}(\overrightarrow{\mathrm{u}}-\overrightarrow{\mathrm{U}}),
\end{array}
$$

where $\nabla^{2}$ is the Laplace operator, and are displacements of solid $\overrightarrow{\mathrm{u}}=(\mathrm{u}, \mathrm{v}, \mathrm{w})$ and liquid $\overrightarrow{\mathrm{U}}=(\mathrm{U}, \mathrm{V}, \mathrm{W})$ respectively, e and $\in$ are the dilatations of solid and liquid; $\mathrm{A}, \mathrm{N}, \mathrm{Q}, \mathrm{R}$ are all poroelastic constants and $\rho_{\mathrm{ij}} \quad(\mathrm{i}, \mathrm{j}=1,2)$ are the mass coefficients following Biot (1956). The poroelastic constants A, N correspond to familiar Lame' constants in purely elastic solid. The coefficient $\mathrm{N}$ represents the shear modulus of the solid. The coefficient $\mathrm{R}$ is a measure of the pressure required on the liquid to force a certain amount of the liquid into the aggregate while total volume remains constant. The coefficient $Q$ represents the coupling between the volume changes of the solid to that of liquid.

We consider three dimensional wave propagation in poroelastic plate such that the displacements of solid $\overrightarrow{\mathrm{u}}=(\mathrm{u}, \mathrm{v}, \mathrm{w}) \quad$ and liquid $\overrightarrow{\mathrm{U}}=(\mathrm{U}, \mathrm{V}, \mathrm{W})$ are expressed in terms of dilatational and vector potentials

$$
\overrightarrow{\mathrm{u}}=\nabla \Phi_{1}+\operatorname{rot} \vec{\Psi}, \quad \overrightarrow{\mathrm{U}}=\nabla \Phi_{2}+\operatorname{rot} \vec{\Psi}^{*}
$$

where

$$
\vec{\Psi}=\left(\Psi_{1}, \Psi_{2}, \Psi_{3}\right), \quad \vec{\Psi}^{*}=\left(\Psi_{1}^{*}, \Psi_{2}^{*}, \Psi_{3}^{*}\right) .
$$

Substituting equation (3) into equation (2), displacements of solid and liquid are

$$
\begin{aligned}
& \mathrm{u}=\frac{\partial \Phi_{1}}{\partial \mathrm{x}}+\frac{\partial \Psi_{3}}{\partial \mathrm{y}}-\frac{\partial \Psi_{2}}{\partial \mathrm{z}}, \quad \mathrm{v}=\frac{\partial \Phi_{1}}{\partial \mathrm{y}}+\frac{\partial \Psi_{1}}{\partial \mathrm{z}}-\frac{\partial \Psi_{3}}{\partial \mathrm{x}}, \quad \mathrm{W}=\frac{\partial \Phi_{1}}{\partial \mathrm{z}}+\frac{\partial \Psi_{2}}{\partial \mathrm{x}}-\frac{\partial \Psi_{1}}{\partial \mathrm{y}}, \\
& \mathrm{U}=\frac{\partial \Phi_{2}}{\partial \mathrm{x}}+\frac{\partial \Psi_{3}^{*}}{\partial \mathrm{y}}-\frac{\partial \Psi_{2}^{*}}{\partial \mathrm{z}}, \quad \mathrm{V}=\frac{\partial \Phi_{2}}{\partial \mathrm{y}}+\frac{\partial \Psi_{1}^{*}}{\partial \mathrm{z}}-\frac{\partial \Psi_{3}^{*}}{\partial \mathrm{x}}, \quad \mathrm{W}=\frac{\partial \Phi_{2}}{\partial \mathrm{z}}+\frac{\partial \Psi_{2}^{*}}{\partial \mathrm{x}}-\frac{\partial \Psi_{1}^{*}}{\partial \mathrm{y}}
\end{aligned}
$$

where $\Phi_{1}, \Phi_{2}, \Psi_{1}, \Psi_{2}, \Psi_{3}, \Psi^{*}{ }_{1}, \Psi^{*}{ }_{2}, \Psi^{*}{ }_{3}$ are functions of $\mathrm{x}, \mathrm{y}, \mathrm{z}$ and time t.

Substitution of equations (2), (3) and (4) into equation (1) yield

$$
\begin{array}{rr}
\mathrm{P} \nabla^{2} \Phi_{1}+\mathrm{Q} \nabla^{2} \Phi_{2}=\frac{\partial^{2}}{\partial \mathrm{t}^{2}}\left(\rho_{11} \Phi_{1}+\rho_{12} \Phi_{2}\right)+\mathrm{b} \frac{\partial}{\partial \mathrm{t}}\left(\Phi_{1}-\Phi_{2}\right), & \mathrm{Q} \nabla^{2} \Phi_{1}+\mathrm{R} \nabla^{2} \Phi_{2}=\frac{\partial^{2}}{\partial \mathrm{t}^{2}}\left(\rho_{12} \Phi_{1}+\rho_{22} \Phi_{2}\right)-\mathrm{b} \frac{\partial}{\partial \mathrm{t}}\left(\Phi_{1}-\Phi_{2}\right), \\
\mathrm{N} \nabla^{2} \Psi_{\mathrm{i}}=\frac{\partial^{2}}{\partial \mathrm{t}^{2}}\left(\rho_{11} \Psi_{\mathrm{i}}+\rho_{12} \Psi_{\mathrm{i}}^{*}\right)+\mathrm{b} \frac{\partial}{\partial \mathrm{t}}\left(\Psi_{\mathrm{i}}-\Psi_{\mathrm{i}}^{*}\right), & 0=\frac{\partial^{2}}{\partial \mathrm{t}^{2}}\left(\rho_{12} \Psi_{\mathrm{i}}+\rho_{22} \Psi_{\mathrm{i}}^{*}\right)-\mathrm{b} \frac{\partial}{\partial \mathrm{t}}\left(\Psi_{\mathrm{i}}-\Psi_{\mathrm{i}}^{*}\right),
\end{array}
$$

where $\mathrm{P}=\mathrm{A}+2 \mathrm{~N}$ and $\mathrm{i}=1,2,3$.

The equation of motion for a homogeneous, isotropic, inviscid elastic fluid is

$$
\nabla^{2} \Phi_{\mathrm{f}}=\frac{1}{\mathrm{~V}_{\mathrm{f}}^{2}} \frac{\partial^{2} \Phi_{\mathrm{f}}}{\partial \mathrm{t}^{2}}
$$

where $\Phi_{\mathrm{f}}$ is displacement potential function and $\mathrm{V}_{\mathrm{f}}$ is the velocity of sound in the fluid. The displacement of fluid is $\overrightarrow{\mathrm{u}}_{\mathrm{f}}=\left(\mathrm{u}_{\mathrm{f}}, \mathrm{v}_{\mathrm{f}}, \mathrm{w}_{\mathrm{f}}\right)$.

The stresses $\sigma_{i j}$ and the liquid pressure s of the poroelastic solid are 


$$
\begin{aligned}
\sigma_{\mathrm{ij}} & =2 \mathrm{Ne}_{\mathrm{ij}}+(\mathrm{Ae}+\mathrm{Q} \in) \delta_{\mathrm{ij}}, \quad(\mathrm{i}, \mathrm{j}=\mathrm{x}, \mathrm{y}, \mathrm{z}), \\
\mathrm{s} & =\mathrm{Qe}+\mathrm{R} \in,
\end{aligned}
$$

where $\delta_{\mathrm{ij}}$ is the well-known Kronecker delta function.

The fluid pressure $\mathrm{P}_{\mathrm{f}}$ is given by

$$
P_{f}=-\rho_{f} \frac{\partial^{2} \Phi_{f}}{\partial t^{2}} .
$$

In equation $(8), \rho_{\mathrm{f}}$ is the density of the fluid.

The dilatations of solid and liquid are

$$
\mathrm{e}=\nabla^{2} \Phi_{1}, \quad \in=\nabla^{2} \Phi_{2} .
$$

Displacement of fluid is given by

$$
\left(u_{f}, v_{f}, w_{f}\right)=\left(\frac{\partial \Phi_{f}}{\partial x}, \frac{\partial \Phi_{f}}{\partial y}, \frac{\partial \Phi_{f}}{\partial z}\right) .
$$

The subscript ' $\mathrm{fl}$ ' or ' $\mathrm{f} 2$ ' associated with a fluid quantity represents that the quantity is related to fluid $\mathrm{fl}$ or fluid f2. For example, $\mathrm{V}_{\mathrm{f} 1}$ is the velocity of sound in the fluid $\mathrm{fl}$ and $\mathrm{P}_{\mathrm{f} 2}$ is the pressure of fluid $\mathrm{f} 2$.

\section{Formulation and solution of the problem}

Let $(\mathrm{x}, \mathrm{y}, \mathrm{z})$ are rectangular co-ordinates. Consider a homogeneous, isotropic, infinite poroelastic plate of thickness $2 \delta$ immersed in an inviscid elastic fluid. The central plane of the plate lie at $\mathrm{z}=0$ and the parallel surfaces of the plate lie at $\mathrm{z}= \pm \delta$. The poroelastic plate is considered to be in contact with fluids on either side. These fluids are denoted by $\mathrm{f} 1$ and $\mathrm{f} 2$. The geometry of the considered problem is:

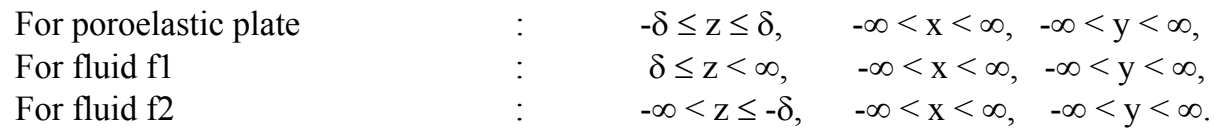

The solution of equation (5) is

$$
\begin{aligned}
& \Phi_{1}=\left[\mathrm{C}_{1} \cos \left(\alpha_{1} \mathrm{z}\right)+\mathrm{C}_{2} \sin \left(\alpha_{1} \mathrm{z}\right)+\mathrm{C}_{3} \cos \left(\alpha_{2} \mathrm{z}\right)+\mathrm{C}_{4} \sin \left(\alpha_{2} \mathrm{z}\right)\right] \cos (n y) \mathrm{e}^{\mathrm{i}(\mathrm{kx}+\omega \mathrm{t})}, \\
& \Phi_{2}=-\left[\mathrm{C}_{1} \delta_{1}^{2} \cos \left(\alpha_{1} \mathrm{z}\right)+\mathrm{C}_{2} \delta_{1}^{2} \sin \left(\alpha_{1} \mathrm{z}\right)+\mathrm{C}_{3} \delta_{2}^{2} \cos \left(\alpha_{2} \mathrm{z}\right)+\mathrm{C}_{4} \delta_{2}^{2} \sin \left(\alpha_{2} \mathrm{z}\right)\right] \cos (n y) \mathrm{e}^{\mathrm{i}(\mathrm{kx}+\omega \mathrm{t})}, \\
& \Psi_{\mathrm{i}}=\left[\mathrm{A}_{\mathrm{i}} \cos \left(\alpha_{3} \mathrm{z}\right)+\mathrm{B}_{\mathrm{i}} \sin \left(\alpha_{3} \mathrm{z}\right)\right] \sin (\mathrm{ny}) \mathrm{e}^{\mathrm{i}(\mathrm{kx}+\omega \mathrm{t})}, \quad \mathrm{i}=1,3, \\
& \Psi_{2}=\left[\mathrm{A}_{2} \cos \left(\alpha_{3} \mathrm{z}\right)+\mathrm{B}_{2} \sin \left(\alpha_{3} \mathrm{z}\right)\right] \cos (\mathrm{ny}) \mathrm{e}^{\mathrm{i}(\mathrm{kx}+\omega \mathrm{t})}, \\
& \Psi_{\mathrm{i}}^{*}=-\frac{\mathrm{K}_{12}}{\mathrm{~K}_{22}} \Psi_{\mathrm{i}}, \quad \mathrm{i}=1,2,3,
\end{aligned}
$$

where $C_{1}, C_{2}, C_{3}, C_{4}, A_{1}, B_{1}, A_{2}, B_{2}, A_{3}, B_{3}$ are constants; $n$, $k$ are wavenumbers, $\omega$ is circular frequency and

$$
\begin{aligned}
& \delta_{\mathrm{i}}^{2}=\frac{1}{\left(\mathrm{RK}_{12}-\mathrm{QK}_{22}\right)}\left[\left(\mathrm{RK}_{11}-\mathrm{QK}_{12}\right)-\mathrm{V}_{\mathrm{i}}^{-2}\left(\mathrm{PR}-\mathrm{Q}^{2}\right)\right], \quad(\mathrm{i}=1,2) \\
& \alpha_{1}^{2}=\frac{\omega^{2}}{\mathrm{~V}_{1}^{2}}-\mathrm{k}^{2}-\mathrm{n}^{2}, \quad \alpha_{2}^{2}=\frac{\omega^{2}}{\mathrm{~V}_{2}^{2}}-\mathrm{k}^{2}-\mathrm{n}^{2}, \quad \alpha_{3}^{2}=\frac{\omega^{2}}{\mathrm{~V}_{3}^{2}}-\mathrm{k}^{2}-\mathrm{n}^{2}, \\
& \mathrm{~K}_{11}=\rho_{11}-\frac{\mathrm{ib}}{\omega}, \quad \mathrm{K}_{12}=\rho_{12}+\frac{\mathrm{ib}}{\omega}, \quad \mathrm{K}_{22}=\rho_{22}-\frac{\mathrm{ib}}{\omega} .
\end{aligned}
$$


In equation (13), $\mathrm{V}_{\mathrm{j}}(\mathrm{j}=1,2)$ are dilatational wave velocities of first and second kind respectively, $\mathrm{V}_{3}$ is shear wave velocity. Similarly, the suitable solution of equation (6) each for fluid $\mathrm{fl}$ and $\mathrm{f} 2$ respectively, is

$$
\Phi_{\mathrm{f} 1}=\mathrm{A}_{4} \cos (\mathrm{ny}) \mathrm{e}^{\mathrm{i}\left(\alpha_{\mathrm{f} 1} z+\mathrm{kx}+\omega \mathrm{t}\right)}, \quad \Phi_{\mathrm{f} 2}=\mathrm{A}_{5} \cos (\mathrm{ny}) \mathrm{e}^{\mathrm{i}\left(-\alpha_{\mathrm{f} z} \mathrm{z}+\mathrm{kx}+\omega \mathrm{t}\right)},
$$

where $\mathrm{A}_{4}, \mathrm{~A}_{5}$ are constants and

$$
\alpha_{\mathrm{f} 1}^{2}=\frac{\omega^{2}}{\mathrm{~V}_{\mathrm{f} 1}^{2}}-\mathrm{k}^{2}-\mathrm{n}^{2}, \quad \alpha_{\mathrm{f} 2}^{2}=\frac{\omega^{2}}{\mathrm{~V}_{\mathrm{f} 2}^{2}}-\mathrm{k}^{2}-\mathrm{n}^{2}
$$

Substituting equation (15) into equation (8), the fluid pressure each for fluid $\mathrm{fl}$ and $\mathrm{f} 2$ is given by

$$
P_{f 1}=\omega^{2} \rho_{f 1} A_{4} \cos (n y) e^{i\left(\alpha_{f 1} z+k x+\omega t\right)}, \quad P_{f 2}=\omega^{2} \rho_{f 2} A_{5} \cos (n y) e^{i\left(-\alpha_{f 2} z+k x+\omega t\right)} .
$$

Similarly employing equation (15) into equation (10) the displacement components each for fluids f1 and f2 are obtained. Substituting equation (11) into equation (4) we obtain the displacements of solid and liquid. With the help of strain displacement relation we find the strain components. Then employing these strains into equation (7), the stresses and liquid pressure in the infinite poroelastic plate are given by

$$
\begin{aligned}
& \sigma_{\mathrm{zz}}=\mathrm{P} \frac{\partial^{2} \Phi_{1}}{\partial \mathrm{z}^{2}}+\mathrm{A}\left(\frac{\partial^{2} \Phi_{1}}{\partial \mathrm{x}^{2}}+\frac{\partial^{2} \Phi_{1}}{\partial \mathrm{y}^{2}}\right)+2 \mathrm{~N}\left(\frac{\partial^{2} \Psi_{2}}{\partial \mathrm{x} \partial \mathrm{z}}-\frac{\partial^{2} \Psi_{1}}{\partial \mathrm{y} \partial \mathrm{z}}\right)+\mathrm{Q} \nabla^{2} \Phi_{2}, \\
& \sigma_{\mathrm{zx}}=\mathrm{N}\left(2 \frac{\partial^{2} \Phi_{1}}{\partial \mathrm{x} \partial \mathrm{z}}+\frac{\partial^{2} \Psi_{3}}{\partial \mathrm{y} \partial \mathrm{z}}-\frac{\partial^{2} \Psi_{1}}{\partial \mathrm{x} \partial \mathrm{y}}-\frac{\partial^{2} \Psi_{2}}{\partial \mathrm{z}^{2}}+\frac{\partial^{2} \Psi_{2}}{\partial \mathrm{x}^{2}}\right), \\
& \sigma_{\mathrm{zy}}=\mathrm{N}\left(2 \frac{\partial^{2} \Phi_{1}}{\partial \mathrm{y} \partial \mathrm{z}}+\frac{\partial^{2} \Psi_{2}}{\partial \mathrm{x} \partial \mathrm{y}}-\frac{\partial^{2} \Psi_{3}}{\partial \mathrm{x} \partial \mathrm{z}}-\frac{\partial^{2} \Psi_{1}}{\partial \mathrm{y}^{2}}+\frac{\partial^{2} \Psi_{1}}{\partial \mathrm{z}^{2}}\right), \\
& \mathrm{s}=\mathrm{Q} \nabla^{2} \Phi_{1}+\mathrm{R} \nabla^{2} \Phi_{2} .
\end{aligned}
$$

The gauge invariance property, following the analysis of Gazis (1959), is used to eliminate two integration constants from equation (12). Without loss of generality of the solution we set $\Psi_{3}=0$ and obtain the required displacement, stresses and liquid pressure.

\section{Boundary conditions - Frequency equation}

For contact between the poroelastic plate and the fluids, we assume that the normal stresses and displacement components in direction of $\mathrm{z}$-axis are continuous at the interfaces $\mathrm{z}=\delta$ and $\mathrm{z}=-\delta$. Thus the boundary conditions in case of a pervious surface are

$$
\begin{aligned}
& \sigma_{\mathrm{zz}}+\mathrm{s}+\mathrm{P}_{\mathrm{f} 1}=0, \quad \sigma_{\mathrm{zx}}=0, \quad \sigma_{\mathrm{zy}}=0, \quad \mathrm{~s}=0, \quad \mathrm{w}-\mathrm{w}_{\mathrm{f} 1}=0, \quad \text { at } \quad \mathrm{z}=\delta, \\
& \sigma_{\mathrm{zz}}+\mathrm{s}+\mathrm{P}_{\mathrm{f} 2}=0, \quad \sigma_{\mathrm{zx}}=0, \quad \sigma_{\mathrm{zy}}=0, \quad \mathrm{~s}=0, \quad \mathrm{w}-\mathrm{w}_{\mathrm{f} 2}=0, \quad \text { at } \quad \mathrm{z}=-\delta \text {. }
\end{aligned}
$$

The boundary conditions in case of an impervious surface are

$$
\begin{aligned}
& \sigma_{\mathrm{zz}}+\mathrm{s}+\mathrm{P}_{\mathrm{f} 1}=0, \quad \sigma_{\mathrm{zx}}=0, \quad \sigma_{\mathrm{zy}}=0, \quad \frac{\partial \mathrm{s}}{\partial \mathrm{z}}=0, \quad \mathrm{w}-\mathrm{w}_{\mathrm{f} 1}=0, \quad \text { at } \mathrm{z}=\delta, \\
& \sigma_{\mathrm{zz}}+\mathrm{s}+\mathrm{P}_{\mathrm{f} 2}=0, \quad \sigma_{\mathrm{zx}}=0, \quad \sigma_{\mathrm{zy}}=0, \quad \frac{\partial \mathrm{s}}{\partial \mathrm{z}}=0, \quad \mathrm{w}-\mathrm{w}_{\mathrm{f} 2}=0, \quad \text { at } \mathrm{z}=-\delta .
\end{aligned}
$$

Substitution of equations (17) and (18) into the equation (19) result in a system of ten homogeneous algebraic equations in ten constants $C_{1}, C_{2}, C_{3}, C_{4}, A_{1}, B_{1}, A_{2}, B_{2}, A_{4}$, and $A_{5}$. For a non-trivial solution, the determinant of the coefficients must vanish. By eliminating these constants, the frequency equation of wave propagation in a poroelastic plate in contact with fluids on either side for a pervious surface is

$$
\left|\mathrm{C}_{\mathrm{ij}}\right|=0, \quad \mathrm{i}, \mathrm{j}=1 \ldots \ldots . .10 .
$$


In equation (21), the elements $\mathrm{C}_{\mathrm{ij}}$ are

$$
\begin{aligned}
& \mathrm{C}_{11}=\left[\left(\alpha_{1}^{2}+\mathrm{k}^{2}+\mathrm{n}^{2}\right)\left(\mathrm{Q} \delta_{1}^{2}-\mathrm{A}+\mathrm{R} \delta_{1}^{2}-\mathrm{Q}\right)-2 \mathrm{~N} \alpha_{1}^{2}\right] \cos \left(\alpha_{1} \delta\right), \\
& \mathrm{C}_{12}=\left[\left(\alpha_{1}^{2}+\mathrm{k}^{2}+\mathrm{n}^{2}\right)\left(\mathrm{Q} \delta_{1}^{2}-\mathrm{A}+\mathrm{R} \delta_{1}^{2}-\mathrm{Q}\right)-2 \mathrm{~N} \alpha_{1}^{2}\right] \sin \left(\alpha_{1} \delta\right), \\
& \mathrm{C}_{13}=\left[\left(\alpha_{2}^{2}+\mathrm{k}^{2}+\mathrm{n}^{2}\right)\left(\mathrm{Q} \delta_{2}^{2}-\mathrm{A}+\mathrm{R} \delta_{2}^{2}-\mathrm{Q}\right)-2 \mathrm{~N} \alpha_{2}^{2}\right] \cos \left(\alpha_{2} \delta\right) \text {, } \\
& \mathrm{C}_{14}=\left[\left(\alpha_{2}^{2}+\mathrm{k}^{2}+\mathrm{n}^{2}\right)\left(\mathrm{Q} \delta_{2}^{2}-\mathrm{A}+\mathrm{R} \delta_{2}^{2}-\mathrm{Q}\right)-2 \mathrm{~N} \alpha_{2}^{2}\right] \sin \left(\alpha_{2} \delta\right), \\
& \mathrm{C}_{15}=2 \mathrm{Nn} \alpha_{3} \sin \left(\alpha_{3} \delta\right), \quad \mathrm{C}_{16}=-2 \mathrm{Nn} \alpha_{3} \cos \left(\alpha_{3} \delta\right), \quad \mathrm{C}_{17}=-2 \mathrm{Nik} \alpha_{3} \sin \left(\alpha_{3} \delta\right), \quad \mathrm{C}_{18}=2 \mathrm{Nik} \alpha_{3} \cos \left(\alpha_{3} \delta\right), \\
& \mathrm{C}_{19}=\rho_{\mathrm{fl}} \omega^{2} \exp \left(\mathrm{i} \alpha_{\mathrm{fl}} \delta\right), \quad \mathrm{C}_{1,10}=0, \quad \mathrm{C}_{21}=-2 \mathrm{Nik} \alpha_{1} \sin \left(\alpha_{1} \delta\right), \quad \mathrm{C}_{22}=2 \mathrm{Nik} \alpha_{1} \cos \left(\alpha_{1} \delta\right), \\
& \mathrm{C}_{23}=-2 \mathrm{Nik} \alpha_{2} \sin \left(\alpha_{2} \delta\right), \quad \mathrm{C}_{24}=2 \mathrm{Nik} \alpha_{2} \cos \left(\alpha_{2} \delta\right), \quad \mathrm{C}_{25}=-\mathrm{Nikncos}\left(\alpha_{3} \delta\right), \quad \mathrm{C}_{26}=-\mathrm{Niknsin}\left(\alpha_{3} \delta\right), \\
& \mathrm{C}_{27}=\mathrm{N}\left(\alpha_{3}^{2}-\mathrm{k}^{2}\right) \cos \left(\alpha_{3} \delta\right), \quad \mathrm{C}_{28}=\mathrm{N}\left(\alpha_{3}^{2}-\mathrm{k}^{2}\right) \sin \left(\alpha_{3} \delta\right), \quad \mathrm{C}_{29}=0, \quad \mathrm{C}_{2,10}=0, \\
& \mathrm{C}_{31}=-2 \mathrm{Nn} \alpha_{1} \sin \left(\alpha_{1} \delta\right), \quad \mathrm{C}_{32}=2 \mathrm{Nn} \alpha_{1} \cos \left(\alpha_{1} \delta\right), \quad \mathrm{C}_{33}=-2 \mathrm{Nn} \alpha_{2} \sin \left(\alpha_{2} \delta\right), \quad \mathrm{C}_{34}=2 \mathrm{Nn} \alpha_{2} \cos \left(\alpha_{2} \delta\right), \\
& \mathrm{C}_{35}=\mathrm{N}\left(\mathrm{n}^{2}-\alpha_{3}^{2}\right) \cos \left(\alpha_{3} \delta\right), \quad \mathrm{C}_{36}=\mathrm{N}\left(\mathrm{n}^{2}-\alpha_{3}^{2}\right) \sin \left(\alpha_{3} \delta\right), \\
& \mathrm{C}_{37}=-\operatorname{Nikncos}\left(\alpha_{3} \delta\right), \quad \mathrm{C}_{38}=\operatorname{Niknsin}\left(\alpha_{3} \delta\right), \quad \mathrm{C}_{39}=0, \quad \mathrm{C}_{3,10}=0, \\
& \mathrm{C}_{41}=\left(\alpha_{1}^{2}+\mathrm{k}^{2}+\mathrm{n}^{2}\right)\left(\mathrm{R} \delta_{1}^{2}-\mathrm{Q}\right) \cos \left(\alpha_{1} \delta\right), \quad \mathrm{C}_{42}=\left(\alpha_{1}^{2}+\mathrm{k}^{2}+\mathrm{n}^{2}\right)\left(\mathrm{R} \delta_{1}^{2}-\mathrm{Q}\right) \sin \left(\alpha_{1} \delta\right), \\
& \mathrm{C}_{43}=\left(\alpha_{2}^{2}+\mathrm{k}^{2}+\mathrm{n}^{2}\right)\left(\mathrm{R} \delta_{2}^{2}-\mathrm{Q}\right) \cos \left(\alpha_{2} \delta\right), \quad \mathrm{C}_{44}=\left(\alpha_{2}^{2}+\mathrm{k}^{2}+\mathrm{n}^{2}\right)\left(\mathrm{R} \delta_{2}^{2}-\mathrm{Q}\right) \sin \left(\alpha_{2} \delta\right), \\
& \mathrm{C}_{45}=0, \quad \mathrm{C}_{46}=0, \quad \mathrm{C}_{47}=0, \quad \mathrm{C}_{48}=0, \quad \mathrm{C}_{49}=0, \quad \mathrm{C}_{4,10}=0 \text {, } \\
& C_{51}=-\alpha_{1} \sin \left(\alpha_{1} \delta\right), \quad C_{52}=\alpha_{1} \cos \left(\alpha_{1} \delta\right), \quad C_{53}=-\alpha_{2} \sin \left(\alpha_{2} \delta\right), \quad C_{54}=\alpha_{2} \cos \left(\alpha_{2} \delta\right), \\
& \mathrm{C}_{55}=-\mathrm{n} \cos \left(\alpha_{3} \delta\right), \quad \mathrm{C}_{56}=\mathrm{n} \sin \left(\alpha_{3} \delta\right), \quad \mathrm{C}_{57}=\mathrm{ik} \cos \left(\alpha_{3} \delta\right), \quad \mathrm{C}_{58}=\mathrm{iksin}\left(\alpha_{3} \delta\right), \\
& \mathrm{C}_{59}=-\mathrm{i} \alpha_{\mathrm{fl}} \exp \left(\mathrm{i} \alpha_{\mathrm{fl}} \delta\right), \quad \mathrm{C}_{5,10}=0, \quad \mathrm{C}_{61}=\left[\left(\alpha_{1}^{2}+\mathrm{k}^{2}+\mathrm{n}^{2}\right)\left(\mathrm{Q} \delta_{1}^{2}-\mathrm{A}+\mathrm{R} \delta_{1}^{2}-\mathrm{Q}\right)-2 \mathrm{~N} \alpha_{1}^{2}\right] \cos \left(\alpha_{1} \delta\right), \\
& \mathrm{C}_{62}=-\left[\left(\alpha_{1}^{2}+\mathrm{k}^{2}+\mathrm{n}^{2}\right)\left(\mathrm{Q} \delta_{1}^{2}-\mathrm{A}+\mathrm{R} \delta_{1}^{2}-\mathrm{Q}\right)-2 \mathrm{~N} \alpha_{1}^{2}\right] \sin \left(\alpha_{1} \delta\right), \\
& \mathrm{C}_{63}=\left[\left(\alpha_{2}^{2}+\mathrm{k}^{2}+\mathrm{n}^{2}\right)\left(\mathrm{Q} \delta_{2}^{2}-\mathrm{A}+\mathrm{R} \delta_{2}^{2}-\mathrm{Q}\right)-2 \mathrm{~N} \alpha_{2}^{2}\right] \cos \left(\alpha_{2} \delta\right) \text {, } \\
& \mathrm{C}_{64}=-\left[\left(\alpha_{2}^{2}+\mathrm{k}^{2}+\mathrm{n}^{2}\right)\left(\mathrm{Q} \delta_{2}^{2}-\mathrm{A}+\mathrm{R} \delta_{2}^{2}-\mathrm{Q}\right)-2 \mathrm{~N} \alpha_{2}^{2}\right] \sin \left(\alpha_{2} \delta\right), \\
& \mathrm{C}_{65}=-2 \mathrm{Nn} \alpha_{3} \sin \left(\alpha_{3} \delta\right), \quad \mathrm{C}_{66}=-2 \mathrm{Nn} \alpha_{3} \cos \left(\alpha_{3} \delta\right), \quad \mathrm{C}_{67}=2 \mathrm{Nik} \alpha_{3} \sin \left(\alpha_{3} \delta\right), \quad \mathrm{C}_{68}=2 \mathrm{Nik} \alpha_{3} \cos \left(\alpha_{3} \delta\right), \\
& \mathrm{C}_{69}=0, \quad \mathrm{C}_{6,10}=\rho_{\mathrm{f} 2} \omega^{2} \exp \left(\mathrm{io}_{\mathrm{f} 2} \delta\right), \\
& \mathrm{C}_{71}=2 \mathrm{Nik} \alpha_{1} \sin \left(\alpha_{1} \delta\right), \quad \mathrm{C}_{72}=2 \mathrm{Nik} \alpha_{1} \cos \left(\alpha_{1} \delta\right), \quad \mathrm{C}_{73}=2 \mathrm{Nik} \alpha_{2} \sin \left(\alpha_{2} \delta\right), \quad \mathrm{C}_{74}=2 \mathrm{Nik} \alpha_{2} \sin \left(\alpha_{2} \delta\right), \\
& \mathrm{C}_{75}=-\mathrm{Nikn \operatorname {cos }}\left(\alpha_{3} \delta\right), \quad \mathrm{C}_{76}=\mathrm{Nikn \operatorname {sin }}\left(\alpha_{3} \delta\right), \quad \mathrm{C}_{77}=\mathrm{N}\left(\alpha_{3}^{2}-\mathrm{k}^{2}\right) \cos \left(\alpha_{3} \delta\right), \quad \mathrm{C}_{78}=-\mathrm{N}\left(\alpha_{3}^{2}-\mathrm{k}^{2}\right) \sin \left(\alpha_{3} \delta\right), \\
& \mathrm{C}_{79}=0, \quad \mathrm{C}_{7,10}=0 \text {, } \\
& \mathrm{C}_{81}=2 \mathrm{Nn} \alpha_{1} \sin \left(\alpha_{1} \delta\right), \quad \mathrm{C}_{82}=2 \mathrm{Nn} \alpha_{1} \cos \left(\alpha_{1} \delta\right), \quad \mathrm{C}_{83}=2 \mathrm{Nn} \alpha_{2} \sin \left(\alpha_{2} \delta\right), \quad \mathrm{C}_{84}=2 \mathrm{Nn} \alpha_{2} \cos \left(\alpha_{2} \delta\right), \\
& \mathrm{C}_{85}=\mathrm{N}\left(\mathrm{n}^{2}-\alpha_{3}^{2}\right) \cos \left(\alpha_{3} \delta\right), \quad \mathrm{C}_{86}=-\mathrm{N}\left(\mathrm{n}^{2}-\alpha_{3}^{2}\right) \sin \left(\alpha_{3} \delta\right), \quad \mathrm{C}_{87}=-\mathrm{Nikncos}\left(\alpha_{3} \delta\right), \quad \mathrm{C}_{88}=-2 \mathrm{Niknsin}\left(\alpha_{3} \delta\right), \\
& \mathrm{C}_{89}=0, \quad \mathrm{C}_{8,10}=0, \\
& \mathrm{C}_{91}=\left(\alpha_{1}^{2}+\mathrm{k}^{2}+\mathrm{n}^{2}\right)\left(\mathrm{R} \delta_{1}^{2}-\mathrm{Q}\right) \cos \left(\alpha_{1} \delta\right), \quad \mathrm{C}_{92}=-\left(\alpha_{1}^{2}+\mathrm{k}^{2}+\mathrm{n}^{2}\right)\left(\mathrm{R} \delta_{1}^{2}-\mathrm{Q}\right) \sin \left(\alpha_{1} \delta\right), \\
& \mathrm{C}_{93}=\left(\alpha_{2}^{2}+\mathrm{k}^{2}+\mathrm{n}^{2}\right)\left(\mathrm{R} \delta_{2}^{2}-\mathrm{Q}\right) \cos \left(\alpha_{2} \delta\right), \quad \mathrm{C}_{94}=-\left(\alpha_{2}^{2}+\mathrm{k}^{2}+\mathrm{n}^{2}\right)\left(\mathrm{R} \delta_{2}^{2}-\mathrm{Q}\right) \sin \left(\alpha_{2} \delta\right), \\
& \mathrm{C}_{95}=0, \quad \mathrm{C}_{96}=0, \quad \mathrm{C}_{97}=0, \quad \mathrm{C}_{98}=0, \quad \mathrm{C}_{99}=0, \quad \mathrm{C}_{9,10}=0,
\end{aligned}
$$




$$
\begin{array}{llll}
\mathrm{C}_{10,1}=\alpha_{1} \sin \left(\alpha_{1} \delta\right), & \mathrm{C}_{10,2}=\alpha_{1} \cos \left(\alpha_{1} \delta\right), & \mathrm{C}_{10,3}=\alpha_{2} \sin \left(\alpha_{2} \delta\right), & \mathrm{C}_{10,4}=\alpha_{2} \cos \left(\alpha_{2} \delta\right), \\
\mathrm{C}_{10,5}=-\mathrm{n} \cos \left(\alpha_{3} \delta\right), & \mathrm{C}_{10,6}=-\mathrm{n} \sin \left(\alpha_{3} \delta\right), & \mathrm{C}_{10,7}=\mathrm{ik} \cos \left(\alpha_{3} \delta\right), & \mathrm{C}_{10,8}=-\mathrm{ik} \sin \left(\alpha_{3} \delta\right), \\
\mathrm{C}_{10,9}=0, & \mathrm{C}_{10,10}=\mathrm{i} \alpha_{\mathrm{f} 2} \exp \left(\mathrm{i} \alpha_{\mathrm{f} 2} \delta\right),
\end{array}
$$

$\mathrm{C}_{10,10}$ represents the element appearing in 10th row and10 column.

Arguing on similar lines, employing the boundary conditions (20) frequency equation of an impervious surface is

$$
\left|D_{i j}\right|=0, i, j=1,2, \ldots . .10 \text {, }
$$

where the elements $\mathrm{D}_{\mathrm{ij}}$ appearing in equation (23) are

$$
\begin{array}{ll}
\mathrm{D}_{\mathrm{ij}}=\mathrm{C}_{\mathrm{ij}} ; & \mathrm{i}=1,2,3,5,6,7,8,10, \quad \mathrm{j}=1,2, \ldots .10, \\
\mathrm{D}_{41}=-\left(\alpha_{1}^{3}+\mathrm{k}^{2} \alpha_{1}+\mathrm{n}^{2} \alpha_{1}\right)\left(\mathrm{R} \delta_{1}^{2}-\mathrm{Q}\right) \sin \left(\alpha_{1} \delta\right), & \mathrm{D}_{42}=\left(\alpha_{1}^{3}+\mathrm{k}^{2} \alpha_{1}+\mathrm{n}^{2} \alpha_{1}\right)\left(\mathrm{R} \delta_{1}^{2}-\mathrm{Q}\right) \cos \left(\alpha_{1} \delta\right), \\
\mathrm{D}_{43}=-\left(\alpha_{2}^{3}+\mathrm{k}^{2} \alpha_{2}+\mathrm{n}^{2} \alpha_{2}\right)\left(\mathrm{R} \delta_{2}^{2}-\mathrm{Q}\right) \sin \left(\alpha_{2} \delta\right), & \mathrm{D}_{44}=\left(\alpha_{2}^{3}+\mathrm{k}^{2} \alpha_{2}+\mathrm{n}^{2} \alpha_{2}\right)\left(\mathrm{R} \delta_{2}^{2}-\mathrm{Q}\right) \cos \left(\alpha_{2} \delta\right), \\
\mathrm{D}_{45}=0, \quad \mathrm{D}_{46}=0, \quad \mathrm{D}_{47}=0, \quad \mathrm{D}_{48}=0, & \mathrm{D}_{49}=0, \quad \mathrm{D}_{4,10}=0, \\
\mathrm{D}_{91}=\left(\alpha_{1}^{3}+\mathrm{k}^{2} \alpha_{1}+\mathrm{n}^{2} \alpha_{1}\right)\left(\mathrm{R} \delta_{1}^{2}-\mathrm{Q}\right) \sin \left(\alpha_{1} \delta\right), & \mathrm{D}_{92}=\left(\alpha_{1}^{3}+\mathrm{k}^{2} \alpha_{1}+\mathrm{n}^{2} \alpha_{1}\right)\left(\mathrm{R} \delta_{1}^{2}-\mathrm{Q}\right) \cos \left(\alpha_{1} \delta\right), \\
\mathrm{D}_{93}=\left(\alpha_{2}^{3}+\mathrm{k}^{2} \alpha_{2}+\mathrm{n}^{2} \alpha_{2}\right)\left(\mathrm{R} \delta_{2}^{2}-\mathrm{Q}\right) \sin \left(\alpha_{2} \delta\right), & \mathrm{D}_{94}=\left(\alpha_{2}^{3}+\mathrm{k}^{2} \alpha_{2}+\mathrm{n}^{2} \alpha_{2}\right)\left(\mathrm{R} \delta_{2}^{2}-\mathrm{Q}\right) \cos \left(\alpha_{2} \delta\right), \\
\mathrm{D}_{95}=0, \quad \mathrm{D}_{96}=0, \quad \mathrm{D}_{97}=0, \quad \mathrm{D}_{98}=0, & \mathrm{D}_{99}=0, \quad \mathrm{D}_{9,10}=0 .
\end{array}
$$

Elements $\mathrm{C}_{\mathrm{ij}}$ appearing in equation (24) are defined in equation (22).

Equation (23) is the frequency equation of three dimensional wave propagation in an infinite poroelastic plate immersed in an inviscid elastic fluid for an impervious surface.

\subsection{Poroelastic plate in contact with fluid}

When the fluid in the domain $-\infty<\mathrm{z} \leq-\delta, \quad-\infty<\mathrm{x}<\infty, \quad-\infty<\mathrm{y}<\infty$, that is, fluid f2 vanish (or $\rho_{\mathrm{f} 2} \rightarrow 0$ ), then the considered problem reduce to a problem of three dimensional wave propagation in poroelastic plate in contact with an inviscid elastic fluid. In this case, the frequency equation of a pervious surface (21) under suitable boundary conditions is reduced to

$$
\left|\mathrm{C}_{\mathrm{ij}}\right|=0, \quad \mathrm{i}, \mathrm{j}=1,2, \ldots \ldots . .9 \text {, }
$$

where the elements $C_{\mathrm{ij}}$ are defined in equation (22) are now evaluated for $\rho_{\mathrm{f} 2}=0$.

Similarly, for $\rho_{\mathrm{f} 2}=0$ equation (23) reduce to

$$
\left|D_{i j}\right|=0, \quad i, j=1,2, \ldots \ldots . .9,
$$

Where the elements $D_{i j}$ appearing in equation (26) are defined in equation (24) are now evaluated for $\rho_{\mathrm{f} 2}=0$.

Equations (25) and (26) are the frequency equations of three dimensional wave propagation in poroelastic plate in contact with an inviscid elastic fluid for a pervious and an impervious surface respectively.

\subsection{Poroelastic plate in vacuum}

When the fluids $\mathrm{f} 1$ and $\mathrm{f} 2$ vanish, that is, $\rho_{\mathrm{f} 1} \rightarrow 0$ and $\rho_{\mathrm{f} 2} \rightarrow 0$ the considered problem reduce to the problem of three dimensional wave propagation in an infinite poroelastic plate in vacuum. Thus equation (21) under suitable boundary conditions reduce to

$$
\left|\mathrm{C}_{\mathrm{ij}}\right|=0, \quad \mathrm{i}=1,2,3,4,6,7,8,9, \quad \mathrm{j}=1,2,3,4,5,6,7,8,
$$

where the elements $C_{i j}$ are defined in equation (22) are now evaluated for $\rho_{\mathrm{f} 1}=0, \rho_{\mathrm{f} 2}=0$.

Similarly, equation (23) under the conditions $\rho_{\mathrm{f} 1}=0, \rho_{\mathrm{f} 2}=0$ reduce to

$$
\left|D_{i j}\right|=0, \quad i=1,2,3,4,6,7,8,9, \quad j=1,2,3,4,5,6,7,8,
$$


where the elements $D_{\mathrm{ij}}$ are defined in equation (24) are now evaluated for $\rho_{\mathrm{f} 1}=0, \rho_{\mathrm{f} 2}=0$.

Equations (27) and (28) are the frequency equations of three dimensional wave propagation in poroelastic plate in vacuum for a pervious and an impervious surface, respectively.

\subsection{Motion having infinite wavelength - Cut-off frequency}

When the wavelength is very long compared to thickness of the poroelastic plate, the wavenumber is zero. The frequencies obtained by equating the wavenumber to zero are referred to as the cut-off frequencies. Thus for $\mathrm{k}=0$, the frequency equation of pervious surface (21) reduce to the product of two determinants

where $\mathrm{D}_{1}$ and $\mathrm{D}_{2}$ are

$$
\mathrm{D}_{1} \mathrm{D}_{2}=0 \text {, }
$$

$$
\mathrm{D}_{1}=\left|\begin{array}{cccccccc}
\mathrm{C}_{11} & \mathrm{C}_{12} & \mathrm{C}_{13} & \mathrm{C}_{14} & \mathrm{C}_{15} & \mathrm{C}_{16} & \mathrm{C}_{19} & 0 \\
\mathrm{C}_{31} & \mathrm{C}_{32} & \mathrm{C}_{33} & \mathrm{C}_{34} & \mathrm{C}_{35} & \mathrm{C}_{36} & 0 & 0 \\
\mathrm{C}_{41} & \mathrm{C}_{42} & \mathrm{C}_{43} & \mathrm{C}_{44} & 0 & 0 & 0 & 0 \\
\mathrm{C}_{51} & \mathrm{C}_{52} & \mathrm{C}_{53} & \mathrm{C}_{54} & \mathrm{C}_{55} & \mathrm{C}_{56} & \mathrm{C}_{59} & 0 \\
\mathrm{C}_{61} & \mathrm{C}_{62} & \mathrm{C}_{63} & \mathrm{C}_{64} & \mathrm{C}_{65} & \mathrm{C}_{66} & 0 & \mathrm{C}_{6,10} \\
\mathrm{C}_{81} & \mathrm{C}_{82} & \mathrm{C}_{83} & \mathrm{C}_{84} & \mathrm{C}_{85} & \mathrm{C}_{86} & 0 & 0 \\
\mathrm{C}_{91} & \mathrm{C}_{92} & \mathrm{C}_{93} & \mathrm{C}_{94} & 0 & 0 & 0 & 0 \\
\mathrm{C}_{10,1} & \mathrm{C}_{10,2} & \mathrm{C}_{10,3} & \mathrm{C}_{10,4} & \mathrm{C}_{10,5} & \mathrm{C}_{10,6} & 0 & \mathrm{C}_{10,10}
\end{array}\right|, \quad \mathrm{D}_{2}=\left|\begin{array}{ll}
\mathrm{C}_{27} & \mathrm{C}_{28} \\
\mathrm{C}_{77} & \mathrm{C}_{78}
\end{array}\right| .
$$

Similarly, equation (23) for infinite wavelength (that is, $\mathrm{k}=0$ ) reduce to the product of two determinants

where $\mathrm{D}_{3}$ and $\mathrm{D}_{4}$ are

$$
\mathrm{D}_{3} \mathrm{D}_{4}=0 \text {, }
$$

$$
\mathrm{D}_{3}=\left|\begin{array}{cccccccc}
\mathrm{D}_{11} & \mathrm{D}_{12} & \mathrm{D}_{13} & \mathrm{D}_{14} & \mathrm{D}_{15} & \mathrm{D}_{16} & \mathrm{D}_{19} & 0 \\
\mathrm{D}_{31} & \mathrm{D}_{32} & \mathrm{D}_{33} & \mathrm{D}_{34} & \mathrm{D}_{35} & \mathrm{D}_{36} & 0 & 0 \\
\mathrm{D}_{41} & \mathrm{D}_{42} & \mathrm{D}_{43} & \mathrm{D}_{44} & 0 & 0 & 0 & 0 \\
\mathrm{D}_{51} & \mathrm{D}_{52} & \mathrm{D}_{53} & \mathrm{D}_{54} & \mathrm{D}_{55} & \mathrm{D}_{56} & \mathrm{D}_{59} & 0 \\
\mathrm{D}_{61} & \mathrm{D}_{62} & \mathrm{D}_{63} & \mathrm{D}_{64} & \mathrm{D}_{65} & \mathrm{D}_{66} & 0 & \mathrm{D}_{6,10} \\
\mathrm{D}_{81} & \mathrm{D}_{82} & \mathrm{D}_{83} & \mathrm{D}_{84} & \mathrm{D}_{85} & \mathrm{D}_{86} & 0 & 0 \\
\mathrm{D}_{91} & \mathrm{D}_{92} & \mathrm{D}_{93} & \mathrm{D}_{94} & 0 & 0 & 0 & 0 \\
\mathrm{D}_{10,1} & \mathrm{D}_{10,2} & \mathrm{D}_{10,3} & \mathrm{D}_{10,4} & \mathrm{D}_{10,5} & \mathrm{D}_{10,6} & 0 & \mathrm{D}_{10,10}
\end{array}\right|, \quad \mathrm{D}_{4}=\left|\begin{array}{ll}
\mathrm{D}_{27} & \mathrm{D}_{28} \\
\mathrm{D}_{77} & \mathrm{D}_{78}
\end{array}\right| .
$$

In equations (30) and (32), the elements $\mathrm{C}_{\mathrm{ij}}, \mathrm{D}_{\mathrm{ij}}$ are defined in equations (22) and (24) are now evaluated for $\mathrm{k}=0$. Equation (29) is satisfied if $\mathrm{D}_{1}=0$, or $\mathrm{D}_{2}=0$. Equation $\mathrm{D}_{1}=0$ give the cut-off frequency of poroelastic plate immersed in fluid for a pervious surface. In a similar way, $\mathrm{D}_{2}=0$ is the frequency equation of shear vibrations of a poroelastic plate immersed in fluid. The frequency equation of shear waves are unaffected by the presence of fluid on either side of the poroelastic plate. From equation (31), it can be seen that $\mathrm{D}_{3}=0$ gives the cut-off frequency of a poroelastic plate immersed in fluid for an impervious surface and $\mathrm{D}_{4}=0$ is the frequency equation of shear waves. From equations (22) and (24), it is clear that $\mathrm{D}_{2}$ and $\mathrm{D}_{4}$ are same. Hence the shear waves are independent of nature of surface also. When simplified, $\mathrm{D}_{2}=0$ or $\mathrm{D}_{4}=0$ reduce to

$$
\omega=V_{3}\left[\frac{q^{2} \pi^{2}}{4 \delta^{2}}+n^{2}\right]^{\frac{1}{2}}, \quad q=1,2,3, \ldots . .
$$

Equation (33) gives the cut-off frequencies of shear vibrations of poroelastic plate of thickness $2 \delta$.

When the wavenumber along $\mathrm{y}$-axis vanish, that is, $\mathrm{n}=0$, the frequency equation of a pervious surface (22) and the frequency equation of an impervious surface (24) are reduced to the product of frequency equations considered by Tajuddin and Ahmed Shah $(2007,2010(c))$. 


\section{Non-dimensionalization of frequency equation}

In a non-dissipative medium for propagating modes wavenumber $\mathrm{k}$ is real. Phase velocity $\mathrm{C}$ is the ratio of frequency to wavenumber, that is, $\mathrm{C}=\omega / \mathrm{k}$. To analyze the frequency equations (21), (23), (25), (26), (27) and (28) it is convenient to introduce the following non-dimensional parameters:

$$
\begin{aligned}
& \mathrm{a}_{1}=\mathrm{PH}^{-1}, \quad \mathrm{a}_{2}=\mathrm{QH}^{-1}, \quad \mathrm{a}_{3}=\mathrm{RH}^{-1}, \quad \mathrm{a}_{4}=\mathrm{NH}^{-1}, \mathrm{~m}_{11}=\rho_{11} \rho^{-1}, \mathrm{~m}_{12}=\rho_{12} \rho^{-1}, \quad \mathrm{~m}_{22}=\rho_{22} \rho^{-1}, \\
& \widetilde{\mathrm{x}}=\left(\mathrm{V}_{0} \mathrm{~V}_{1}^{-1}\right)^{2}, \quad \widetilde{\mathrm{y}}=\left(\mathrm{V}_{0} \mathrm{~V}_{2}^{-1}\right)^{2}, \quad \widetilde{\mathrm{z}}=\left(\mathrm{V}_{0} \mathrm{~V}_{3}^{-1}\right)^{2}, \quad \beta=\mathrm{k} \delta, \quad \alpha=\mathrm{nk}^{-1}, \quad \Omega=\omega \delta \mathrm{C}_{0}^{-1}, \\
& \mathrm{t}_{0}=\mathrm{n} \delta, \quad \mathrm{t}_{1}=\rho_{\mathrm{f} 1} \rho^{-1}, \quad \mathrm{~m}_{1}=\mathrm{V}_{\mathrm{f} 1} \mathrm{~V}_{3}^{-1}, \quad \mathrm{t}_{2}=\rho_{\mathrm{f} 2} \rho^{-1}, \quad \mathrm{~m}_{2}=\mathrm{V}_{\mathrm{f} 2} \mathrm{~V}_{3}^{-1}, \quad \xi=\mathrm{CC}_{0}^{-1},
\end{aligned}
$$

where $\xi$ is non-dimensional phase velocity, $\beta$ is propagation constant, $\alpha$ is ratio of wavenumbers, $\Omega$ is non-dimensional frequency, $\mathrm{H}=\mathrm{P}+2 \mathrm{Q}+\mathrm{R}, \quad \rho=\rho_{11}+2 \rho_{12}+\rho_{22}, \mathrm{C}_{0}, \mathrm{~V}_{0}$ are the reference velocities $\left(\mathrm{C}_{0}{ }^{2}=\mathrm{N} / \rho, \mathrm{V}_{0}{ }^{2}=\mathrm{H} / \rho\right)$ and $2 \delta$ is thickness of the poroelastic plate.

\section{Numerical results and discussion}

Two types of poroelastic materials namely, sandstone saturated with kerosene (Fatt, 1959) and sandstone saturated with water (Yew and Jogi, 1976) are considered to carry out the computational work. Sandstone saturated with kerosene is designated as Material-I (Mat-I) and sandstone saturated with water is designated as Material-II (Mat-II). Non-dimensional physical parameters of Material-I and II are given in Table-I.

Table - I

\begin{tabular}{|c|c|c|c|c|c|c|c|c|c|c|}
\hline $\begin{array}{c}\text { Material/ } \\
\text { Parameter }\end{array}$ & $\mathrm{a}_{1}$ & $\mathrm{a}_{2}$ & $\mathrm{a}_{3}$ & $\mathrm{a}_{4}$ & $\mathrm{~m}_{11}$ & $\mathrm{~m}_{12}$ & $\mathrm{~m}_{22}$ & $\widetilde{\mathrm{x}}$ & $\widetilde{\mathrm{y}}$ & $\widetilde{\mathrm{z}}$ \\
\hline $\mathrm{I}$ & 0.843 & 0.065 & 0.028 & 0.234 & 0.901 & -0.001 & 0.101 & 0.999 & 4.763 & 3.851 \\
\hline II & 0.960 & 0.006 & 0.028 & 0.412 & 0.877 & 0 & 0.123 & 0.913 & 4.347 & 2.129 \\
\hline
\end{tabular}

Frequency equations of three dimensional wave propagation of poroelastic plate immersed in fluid for a pervious surface (46) and an impervious surface (48), constitute a relation between non-dimensional phase velocity $\xi$ and propagation constant $\beta$ for given physical parameters and for fixed value of ratio of wavenumbers, that is $\alpha$. Non-dimensional phase velocity $\xi$ is determined for different values of $\beta$ each for a pervious and an impervious surface for fixed values of $\alpha$ taken as $\alpha=1,2$. The values of $\beta$ are taken in the interval $[5,10]$ in steps of 0.5 . When $\mathrm{k}=0$, the values of $\mathrm{t}_{0}$ are taken in the interval $[5,10]$ in steps of 0.5 to find the non-dimensional frequency. For poroelastic plate immersed in an inviscid elastic fluid (fluids f1 and f2 are same) or a poroelastic plate in contact with an inviscid elastic fluid, the values of $\mathrm{m}_{1}, \mathrm{~m}_{2}, \mathrm{t}_{1}$ and $\mathrm{t}_{2}$ are taken as $\mathrm{m}_{1}=\mathrm{m}_{2}=6.5$ and $\mathrm{t}_{1}=\mathrm{t}_{2}=0.4$. Phase velocity as a function of propagation constant is presented in fig.1-3 and fig.4-6 for poroelastic plate immersed in an elastic fluid, in contact with fluid and poroelastic plate in vacuum each for a pervious and an impervious surface and for the considered materials when $\alpha=1$ and $\alpha=2$, respectively.
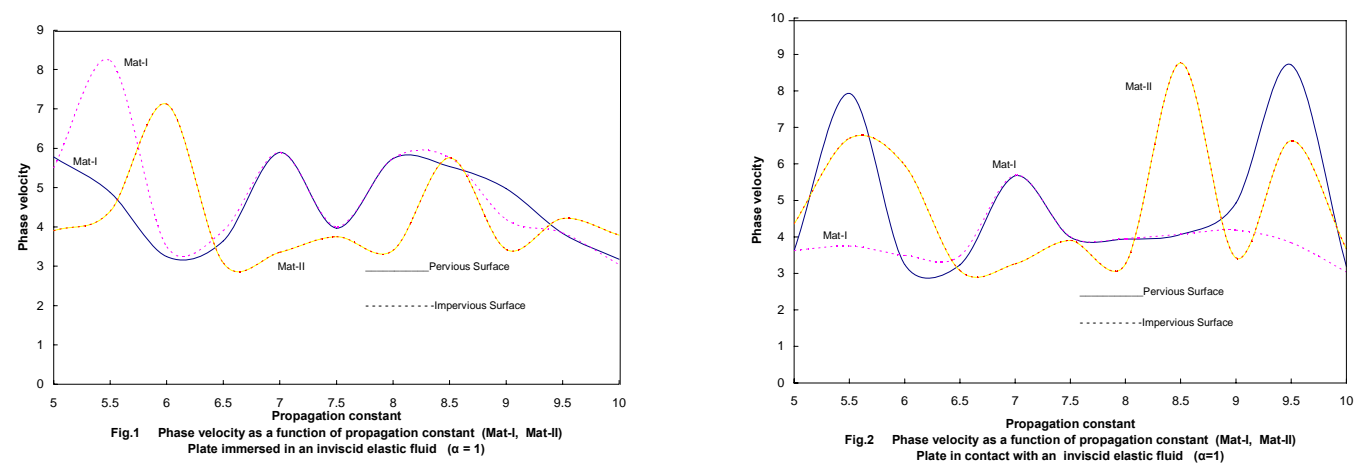

Fig. 1 shows the phase velocity of poroelastic plate immersed in an elastic fluid when $\alpha=1$. From fig. 1 it is seen that the phase velocity of an impervious surface is higher than that of a pervious surface in case of Material-I in $5 \leq \beta \leq 6$. Otherwise, phase velocity of pervious and impervious surface is same. Phase velocity of pervious and impervious surface is same for Material-II. 
Phase velocity of Material-II is less than that of phase velocity of Material-I each for a pervious and an impervious surface in $6.5 \leq \beta \leq 8.5$. Fig. 2 shows the phase velocity of poroelastic plate in contact with an elastic fluid when $\alpha=1$. From fig. 2 it is seen that the phase velocity of pervious and impervious surface is same for Material-II while the phase velocity of a pervious surface is higher than that of impervious surface for Material-I in $5 \leq \beta \leq 6$ and $8.5 \leq \beta \leq 10$. Again phase velocity of Material-II is less than that of Material-I in $6.5 \leq \beta \leq 8$. Phase velocity of a poroelastic plate in vacuum is presented in fig. 3 for the considered materials each for a pervious and an impervious surface when $\alpha=1$.
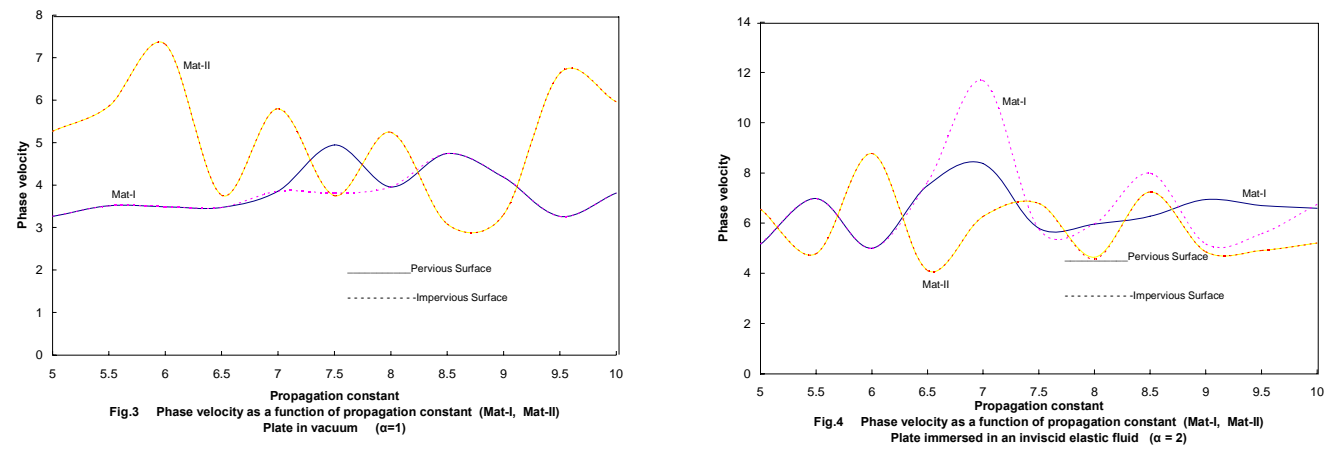

From fig. 3 it is seen that phase velocity of a pervious surface is higher than that of an impervious surface in $7 \leq \beta \leq 8$ in case of Material-I. Otherwise the phase velocity of pervious and impervious surface is same. Phase velocity of pervious and impervious surface is same in case of Material-II. Phase velocity of Material-II is higher than that of Material-I each for a pervious and an impervious surface in $5 \leq \beta<7.5$.
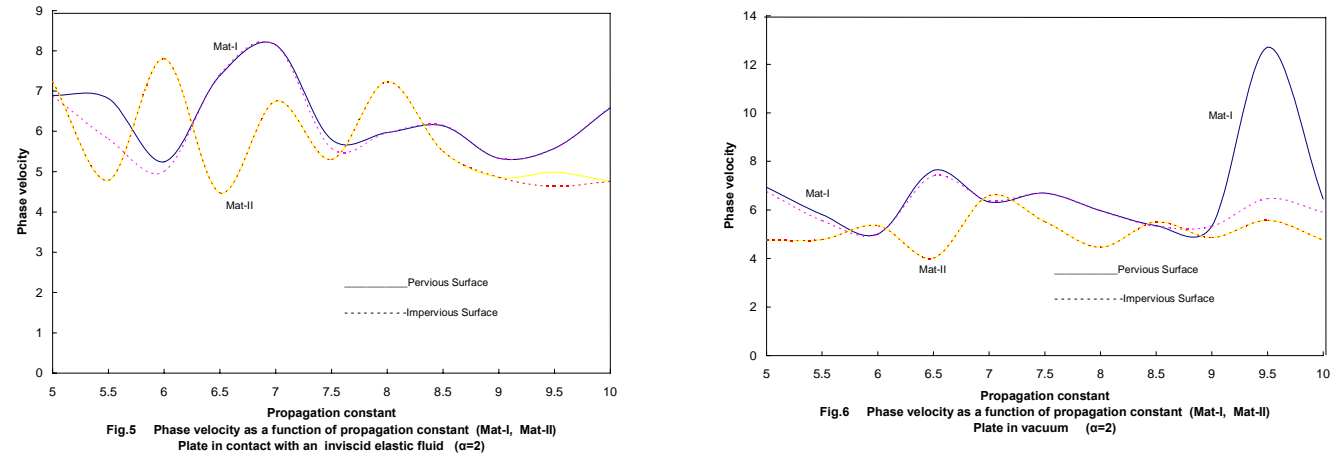

Fig. 4 shows the phase velocity of poroelastic plate immersed in an elastic fluid when $\alpha=2$. From fig. 4 it is seen that the phase velocity of pervious and impervious surface is same for Material-I $5 \leq \beta<6.5$ otherwise, phase velocity of pervious surface is different from that of an impervious surface. Phase velocity of pervious and impervious surface is same for Material-II. Also, it is observed that, in general, the phase velocity each for a pervious and an impervious surface is higher when $\alpha=2$ than that of the phase velocity when $\alpha=1$, for the considered materials. The variation of phase velocity of a poroelastic plate in contact with fluid for the considered materials is shown in fig. 5 when $\alpha=2$. From fig. 5 it is seen that phase velocity of a pervious surface is higher than that of an impervious surface in $5 \leq \beta<6.5$ in case of Material-I otherwise, it is same. Similarly, the phase velocity of pervious and impervious surface is same in case of Material-II. Here too, in general, the phase velocity each for a pervious and an impervious surface is higher when $\alpha=2$ than that of the phase velocity when $\alpha=1$. Variation of phase velocity of a poroelastic plate in vacuum for $\alpha=2$ is presented in fig. 6 for the considered materials. From fig. 6 it is seen that the phase velocity of a pervious surface is higher than that of an impervious surface in $9 \leq \beta \leq 10$ in case of Material-I otherwise, it is same. The phase velocity of Material-II is same for pervious and impervious surfaces. Also the phase velocity of Material-II is less than that of Material-I each for a pervious and an impervious surface. Here it is observed that the phase velocity of in case of Material-II is higher for $\alpha=2$ than that of $\alpha=1$, while this is not true for Material-I. Frequency as a function of $t_{0}$ is presented in fig.7-9 for poroelastic plate immersed in an elastic fluid, in contact with fluid and poroelastic plate in vacuum each for a pervious and an impervious surface and for the considered materials when $\mathrm{k}=0$. From fig. 7 it is seen that there is a gradual increase in frequency with the increase of the values of t0 for both the considered materials for a poroelastic plate immersed in fluid. The frequency of pervious and impervious surface is same each for the considered materials. The frequency of Material-I is higher than that of Material-II each for a pervious and an impervious surface. 

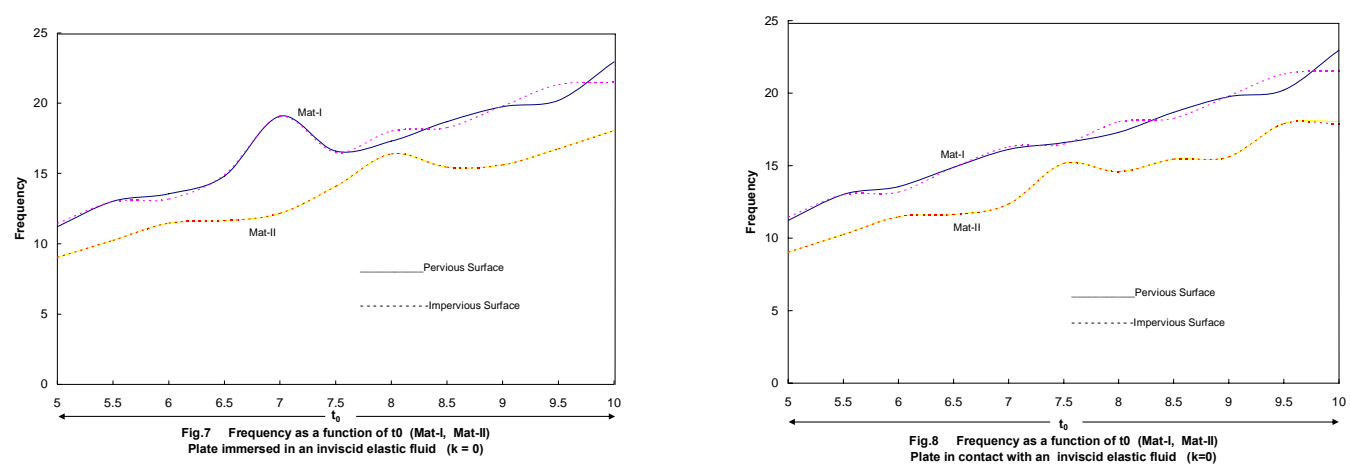

Similar trend is observed in the variation of frequency of poroelastic plate in contact with fluid (see fig.8) and poroelastic plate in vacuum (see fig.9). In fact, the frequency of poroelastic plate in contact with fluid and poroelastic plate in vacuum is nearly same for the considered materials each for a pervious and an impervious surface and it can be said that the presence of fluid on one side of the poroelastic plate has no significant effect on frequency. This is not true in case of a poroelastic plate immersed in elastic fluid.

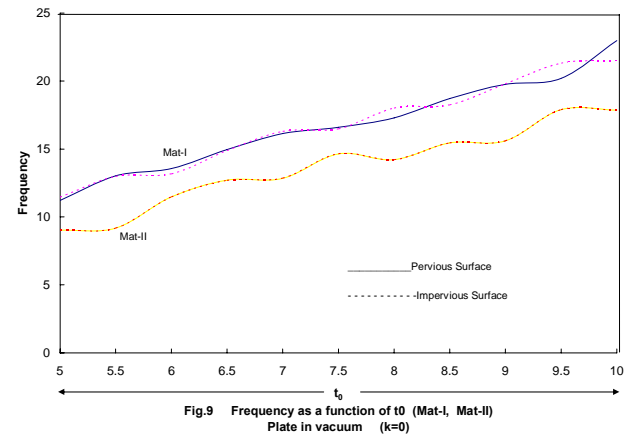

\section{Concluding remarks}

The study of three dimensional wave propagation in poroelastic plate immersed in fluid, poroelastic plate in contact with fluid and poroelastic plate in vacuum has lead to following conclusions:

(i) Phase velocities of pervious and impervious surface is same for Material-II for each of the three considered cases, that is, poroelastic plate immersed in fluid, poroelastic plate in contact with fluid and poroelastic plate in vacuum.

(ii) Phase velocity of the considered materials each for a pervious and an impervious is less when $\alpha=1$ than that of when $\alpha=2$.

(iii) Dilatational and shear waves of a poroelastic plate immersed in fluid are uncoupled when both the wavenumbers vanish.

(iv) Shear waves are independent of nature of surface and presence of fluids on either side of the poroelastic plate.

(v) When the wavenumber $\mathrm{k}$ is zero, the frequency of Material-II is less than that of Material-I for all the considered cases.

(vi) When the wave number $\mathrm{k}$ vanishes, the presence of fluid on one side of the poroelastic plate has no significant effect on frequency.

\section{References}

Banghar, A.R., Murty, G.S., and Raghavacharyulu, I.V.V., 1976. On the parametric model of loose bonding of elastic half spaces, J.Acoust.Soc.Am., Vol. 60, pp.1071-1078.

Biot, M.A., 1956. Theory of propagation of elastic waves in fluid-saturated porous solid, J.Acoust.Soc.Am., vol.28, pp.168-178.

Chao, G., Smeulders, D.M.J., and van Dongen, M.E.H., 2004. Shock-induced borehole waves in porous formations: Theory and experiments, J.Acoust.Soc.Am., Vol. 116, pp. 693-702.

Fatt, I., 1959. The Biot-Willis elastic coefficients for a sandstone, J.Appl.Mech., Vol. 26, pp.296-297.

Gazis, D.C., 1959. Three-dimensional investigation of the propagation of waves in hollow circular cylinders, I., Analytical foundation, J.Acoust.Soc.Am., Vol.31, pp.568-573.

Kanj, M., and Abousleiman, Y., 2004. The Generalized Lame' Problem - Part I: Coupled Poromechanical Solutions, Trans.ASME, J.Appl.Mech., Vol.71, pp.168-179.

Tajuddin, M., and Ahmed, S.I., 1991. Dynamic interaction of a poroelastic layer and a half-space, J.Acoust.Soc.Am., Vol. 89, pp.1169-1175. 
Malla Reddy, P., and Tajuddin, M., 2003. Edge waves in poroelastic plate under plane-stress conditions, J.Acoust.Soc.Am., Vol. 114, pp.185-193.

Mindlin, R.D., and Fox, E.A., 1960. Vibrations and waves in elastic bars of rectangular cross section, J.Appl.Mech., vol.27, pp.152-158.

Mindlin, R.D., 1986. Flexural vibrations of rectangular plates with free edges, Mechanics Research Communications, vol.13, pp.349-357.

Shah, S.A., 2008. Axially symmetric vibrations of fluid-filled poroelastic circular cylindrical shells, Journal of Sound and Vibration, Vol.318, pp.389-405.

Shah, S.A., 2010(a) Flexural wave propagation in coated poroelastic cylinders with reference to fretting fatigue, Journal of Vibration and Control.(In print).

Shah, S.A., and Tajuddin, M., 2009. Axially symmetric vibrations of finite composite poroelastic cylinders, International Journal Applied Mechanics and Engineering, Vol.14, pp. 865-877.

Shah, S.A., and Tajuddin, M., 2010(b). On flexural vibrations of poroelastic circular cylindrical shells immersed in an acoustic medium, Special Topics and Reviews in Porous Media - An International Journal, Vol.1, pp.67-78.

Shah, S.A., and Tajuddin, M., 2010(c). Dispersion of waves in an infinite poroelastic plate immersed in an inviscid elastic fluid, Special Topics and Reviews in Porous Media - An International Journal, Vol.1, pp.269-278.

Shuyu, L., 2001. Study on the flexural vibration of rectangular thin plates with free boundary conditions, Journal of Sound and Vibration., Vol.239, pp.1063-1071.

Tajuddin, M., and Ahmed Shah, S., 2006. Circumferential waves of infinite hollow poroelastic cylinders, Trans.ASME, J.Appl.Mech., Vol.73, pp.705-708.

Tajuddin, M., and Ahmed Shah, S., 2007. On torsional vibrations of infinite hollow poroelastic cylinders, Journal of Mechanics of Materials and Structures, Vol.2, pp.189-200.

Tajuddin, M., and Ahmed Shah, S., 2010. Radial vibrations of thick-walled hollow poroelastic cylinders, Journal of Porous Media, Vol.13, pp.307-318.

Walter, W.W., and Anderson, G.L., 1970. Wave propagation in an infinite elastic plate in contact with an inviscid liquid layer, J.Acoust.Soc.Am., Vol.47, pp.1398-1407.

Yew, C.H. and Jogi, P.N., 1976. Study of wave motions in fluid-saturated porous rocks, J.Acoust.Soc.Am., Vol. 60, pp. 2-8.

\section{Biographical notes}

Dr. S. Ahmed Shah is professor in the Department of Mathematics, Deccan College of Engineering and Technology Hyderabad, India. He has more than 19 years of teaching experience. He published papers in referred national and international journals and has more than 11 years of research experience. He attended and presented papers in national and international conferences. His area of research includes stress wave propagation in poroelastic solids.

Late Dr.M.Tajuddin was a Professor in the Department of Mathematics Osmania University, Hyderabad, India. He had more than 30 years of experience in teaching and research. His area of research includes wave phenomena aspects in elastic porous media. He had published more than forty papers in referred national and international journals. He had also presented several research articles in national and international conferences.

Received September 2010

Accepted March 2011

Final acceptance in revised form March 2011 